\title{
Review on Mechanisms, Importance of Homeostasis and Fluid Imbalances in the Elderly
}

\author{
${\text { AGGIE } \text { BAK }^{1 *} \text { and AMALIA TSIAMI }}^{2}$ \\ ${ }^{1}$ Richard Wells Centre, College of Nursing, Midwifery and Healthcare, \\ University of West London, London. \\ ${ }^{2}$ London Geller College of Hospitality and Tourism, University of West London, London. \\ http://dx.doi.org/10.12944/CRNFSJ.4.Special-Issue-Elderly-November.01
}

(Received: August 22, 2016; Accepted: September 29, 2016)

\begin{abstract}
Water is considered an essential nutrient, but very often is overlooked in favour of nutrition. Due to age related changes, older people are more vulnerable to both, dehydration and overhydration. This article describes the principles of water homeostasis and provides a description of common age-related physiological changes related to water balance. Fluid homeostasis largely depends on osmo- and baro-receptors that respectively react to changes in osmolality and blood pressure, water-regulating hormone release, the subsequent response of kidneys to the hormonal stimulation and activation of thirst. These mechanisms diminish with age and put elderly at risk of dehydration. All involved in a care for the elderly should be informed and become vigilant to prevent dehydration from occurring.
\end{abstract}

Keywords: Homeostasis, Fluid balance, Hydration, Dehydration, Older Adults, Aging.

\section{INTRODUCTION}

Water is the most essential and yet often forgotten nutrient. While undernutrition has been a recognised problem for many years and many recent interventions aimed to raise awareness and improve nutritional status; hydration requirements have often been forgotten 1 . This apparent lack of interest is reflected in the literature as there are few recent epidemiological studies describing incidence or prevalence of dehydration in any populations and elderly in particular. The latest evidence that the problem exists is the publication by Wolff et al (2015) ${ }^{2}$ reporting that the prevalence of hypernatremia in the United Kingdom may be as much as ten-fold higher in elderly patients admitted from care homes than from community. The care home residents described in this study were on average six years older and more likely to suffer from dementia. Hypernatremia is almost exclusively a result of chronic dehydration and has been long regarded as indicator of neglect ${ }^{3}$. Frequent reports by media have recently highlighted the issue of dehydration, which has now gained momentum and is seen as an important aspect in maintaining health and wellbeing in all age groups. While fluid imbalances also include overhydration; dehydration is more often highlighted in the literature and usually perceived as greater threat in older population.

\section{Water homeostasis}

Water homeostasis is largely dependent on fluid intake and output and is controlled by the action of baroreceptors and osmoreceptors signalling via different pathways ${ }^{4}$. In the healthy young adults, reduced blood volume is recognised by the baroreceptors and triggers a cascade of reactions known as renin-angiotensin system ${ }^{5}$. The end products of the cascade have a direct impact on water preservation with aldosterone and angiotensin II acting on kidneys to preserve sodium and facilitating osmotic reabsorption of water ${ }^{6}$. At the same time, antidiuretic hormone $(\mathrm{ADH}$, also known as arginine vasopressin) causes vasoconstriction 
and acts on kidneys to preserve water ${ }^{6}$. In a similar fashion, the osmoreceptors detect a change in osmotic pressure (concentration of solutes in the blood) and act on hypothalamus to increase the secretion of $\mathrm{ADH}^{4}$. Renal conservation, while very efficient in correcting small changes in the blood volume and concentration is not reliable as a sole mechanism of fluid balance because it can only prevent further loss ${ }^{7}$. Daily water intake is therefore necessary to replenish the fluids; this process is triggered by sensation of thirst. This basic behaviour of seeking out water helps restore a fluid balance. Both, osmoreceptors and baroreceptors have an ability to act on central nervous system to trigger the need for fluids ${ }^{4}$. Once the water has been consumed, the sensation diminishes quickly following the oropharyngeal cues and gastric distention ${ }^{4}$.

Human body also has an ability to control the excess of fluids in response to increased blood volume by production of atrial natriuretic hormone (ANP). ANP, released by the cardiac muscle cells has an opposite effect to that observed by $\mathrm{ADH}^{8}$. Release of ANP triggers a response on kidneys to excrete excess water and sodium and inhibit renin secretion and acts on adrenal cortex to reduce the amount of aldosterone secretion ${ }^{5}$.

When the fluid is ingested or reabsorbed from kidneys, it first goes into the blood, but it is quickly distributed evenly between interstitial spaces and plasma ${ }^{5}$. Pumping action of the heart creates hydrostatic pressure that forces the water out of capillaries into the surrounding interstitial space, while osmotic pressure created by solutes diffusing through capillaries into the bloodstream forces the water back. This creates a continuous process of fluid exchange between interstitial and intravascular spaces that aims but never reaches equilibrium ${ }^{5}$. When fluid is in deficit, the blood volume decreases and osmotic pressure increases, causing the shift of water from interstitial to intravascular space. In a similar fashion, to maintain osmolality of extracellular fluid (ECF), the fluid from intracellular (ICF) compartment is shifted into the interstitial space leading to dehydration of the cells ${ }^{5}$. The process is reversed when water is ingested. Additionally, there is some evidence that in times where water is not available, the body also has an ability to draw additional fluid reserves from intracellular fluid compartments in order to defend a vascular tree. The process is supported by retaining plasma albumin, which allows the fluid shift from other places such as gastrointestinal tract ${ }^{9}$. The mechanism has been demonstrated in animal models where fluid depletion led to preferential dehydration of visceral organs such as skin or muscles in order to maintain fluid volume in plasma and preserve vital organs such as brain and liver ${ }^{9,10}$. This emergency system however, is short acting and may have further consequences on health if fluid is not replenished. This is because water is also needed for many intracellular functions without which metabolic processes are hindered ${ }^{11}$. It is also evident that when plasma osmolality is elevated, the brain is able to regain its equilibrium of ICF after initial period of imbalance by producing the osmotically active substances, which appear in the brain tissue within a couple of hours of dehydration occurring ${ }^{10}$. Sodium, the main ion with osmotic activity in extracellular fluid is known to be difficult to correct when raised. Hypernatremia is associated with increased risk of mortality ${ }^{3,8,12}$ and death often occurs following the partial restoration of the water and sodium imbalance, suggesting that too rapid correction of dehydration may have some toxic effects ${ }^{12}$. This occurrence could possibly be the result of the brain absorbing excess water from the blood, leading to swelling and subsequent damage; although this theory would need to be supported by further studies.

\section{Fluid imbalance disorders}

Three potentially life threatening conditions are the result of fluid imbalances of which two relate to water deficit and one to water overload. Classically, the academic literature describes two forms of water deficit: dehydration described as water loss from intracellular volume compartment and hypovolemia due to water losses of extracellular volume ${ }^{13}$. In clinical practice dehydration often relates to both terms and no attempt is made to distinguish the two. Dehydration in clinical setting is commonly described as a decline of total body water (TBW) from any compartments that may or may not be accompanied by electrolyte losses. Clinical dehydration may then be further classified as hypertonic (true physiological dehydration), hypotonic or rarely isotonic (both related to hypovolemia rather than dehydration) ${ }^{13}$. Hypertonic dehydration occurs due to reduced fluid intakes or increased insensible losses via skin 
and respiratory tract, while hypotonic and isotonic dehydration are more commonly result of vomiting and diarrhoea. Consequences also differ depending on the amount of electrolytes lost. Hypertonic dehydration usually leads to neurological changes such as headaches or confusion, decreased or concentrated urine, tiredness and loss of appetite ${ }^{14}$. The hallmark of hypertonic dehydration is also a thirst sensation, a safety mechanism that signals the need for more fluid ${ }^{14}$. If severe, seizures and death may follow ${ }^{14}$. Hypotonic and isotonic dehydration are associated with reduced blood volume therefore orthostatic hypotension, dizziness and fainting is likely followed by hypovolemic shock and death in more severe cases ${ }^{14}$. Thirst would not be present since the mechanism would not be activated in the lack of hyperosmolar state ${ }^{14}$.

Water intoxication or water poisoning is a consequence of the fluid overconsumption without appropriate replacement of the electrolytes. This results in dilution of the solutes and subsequent hypoosmolality. The accidental ingestion is rare, but overhydration has been described in academic literature in endurance athletes consuming hypotonic fluids ${ }^{15}$ and MDMA users ${ }^{16}$. Media have also demonstrated isolated incidences of water intoxication during beverage drinking competitions ${ }^{17,18}$ and most recently while 'detoxing'19. The resulting fluid uptake into intracellular spaces causes the swelling of the cells. In central nervous system swelling induces intracranial pressure and opening of the blood brain barrier resulting in a wide range of neurological changes such as headache and confusion ${ }^{5}$. Fluid uptake into other parts of the body results in nausea, vomiting and bradycardia. In extreme cases, cerebral oedema, seizures and death may occur ${ }^{20}$.

\section{Physiological changes and the risk of water and electrolyte imbalances in the elderly}

Elderly are a particularly vulnerable group of people and are predisposed to the risk of water and electrolyte imbalances. Many physiological changes that could predispose to fluid imbalances have been observed in apparently healthy elderly subjects. The problem may be further complicated by underlying disease that may accelerate the fluid losses or prevent the subjects from obtaining fluids in sufficient amounts to restore the water balance.

\section{Changes in kidney function}

It is well known that human kidneys usually start deteriorating at the age of 30 and up to the age of 90 there could be as much as $30-50 \%$ of the nephron loss ${ }^{21}$. The remaining nephrons perform less sufficiently and their rate of filtration decreases by $10 \%$ every decade, while creatinine clearance may decline by as much as $30 \%$ by the age of $80^{4}$. This explains the observed increased baseline serum creatinine in the elderly subjects ${ }^{22}$. The above changes have a direct impact on the ability to reabsorb solutes and water, making the process of urine concentration largely impaired, leading to excessive water and sodium loss and potentially predisposing to dehydration ${ }^{23}$. On the other hand, due to decreased number of nephrons, the glomerular filtration rate decreases as well, which means that smaller amounts of blood are filtered at the time and that the waste products are eliminated less efficiently ${ }^{23}$. If water was to be consumed in excess, this could lead to accumulation of the fluids in the interstitial space and dilution of the solutes in the blood. Therefore due to the nephron loss and resulting diminished kidney function, elderly subjects have very narrow margins to sustain water homeostasis, which predisposes them to both, under and overhydration.

\section{Hormonal changes}

The diminished kidney results in a diminished renin production, a molecule of which an enzymatic action results in a first step of conversion of angiotensinogen into an active angiotensin. As a consequence, both angiotensin and aldosterone levels are diminished ${ }^{24}$. Elderly have also been found to have increased levels of ADH. This is thought to be a compensatory mechanism since it has also been observed that renal sensitivity to $\mathrm{ADH}$ seems to be impaired $^{25}$.

Furthermore, the 24-hr diuresis rhythm also seems to be affected. The rhythm initially develops in the first years of life and adjusts for excretion of large amounts of urine during the day and smaller amounts at night. This is controlled by the circulating levels of $A D H$, increasing at night to allow the urine output to be below the capacity of the bladder and preventing nocturia ${ }^{26}$. Whereas the younger body produces about $14 \%$ of the urine 
during the night, some elderly excrete about a half or in extreme cases the volume may exceed $85 \%$ during this time ${ }^{27}$. This phenomenon is a result of disturbances in ADH production and subsequent deterioration of the diuresis rhythm. For those with nocturnal polyuria syndrome, the night production of $\mathrm{ADH}$ may decrease to undetectable levels ${ }^{27}$. This has an impact on quality of life due to disturbances in sleep, incontinence and the increased risk of falls ${ }^{28,29}$. Some elderly report increased thirst during the night but there is a concern that many actively restrict fluid intakes to prevent nocturia and incontinence ${ }^{30}$. However, considering that $\mathrm{ADH}$ levels are low, this action has no effect on diuresis ${ }^{31}$ and they may unnecessarily put themselves at risk of dehydration.

\section{Failure to restore euhydration and changes in thirst perception}

It is also well documented that ability to restore water balance and the perception of thirst in the apparently healthy elderly are largely diminished. In the study by Phillips et al (1984) ${ }^{21}$, of 14 healthy young and old men subjected to $24 \mathrm{hr}$ of water deprivation and dry diet; it was observed that despite identical weight loss, indicative of the similar degree of water loss, elderly subjects showed more significant increases in both, blood sodium concentration and osmolality than that observed in younger subjects. Furthermore, following dehydration and free access to water made available to all, it was observed that young subjects consumed enough fluids to improve their hydration status; while elderly subjects, despite the obvious need to consume more water, reported less thirst, drunk less and failed to restore their blood parameters to the baseline values. The authors noted that the hydration status is likely to be restored eventually, but this delayed response could potentially have detrimental effects in some situations. Since the osmotic pressure was higher in the elderly subjects, it would be expected that the thirst would be greater in this group unless the pathways leading to its stimulation were diminished. The impaired thirst response has been confirmed in another study by the same group ${ }^{32}$ where water deprivation for seven hours did not result in changes in thirst ratings in the elderly despite the rising levels of blood sodium and osmolality. Young men acting as controls in this study reported significantly increased thirst within five hours of consuming the last drink.
Similar responses have been observed following dehydration due to heat stress. In the study by Miescher and Fortney (1989) ${ }^{33}$, six young and five healthy older participants were subjected to a hot and dry environment for four hours $\left(42^{\circ} \mathrm{C}, 25 \%\right.$ humidity). No fluids were provided for the first three hours, after which time cool water was available for the remaining 60 minutes. Following the heat exposure, the subjects also completed a 30 minute resting period at $25^{\circ} \mathrm{C}$. Authors have noted that despite the similar rates of sweating observed, elderly subjects' core temperature increased to higher levels than that observed in younger men, suggesting that thermoregulation must have been impaired in the older subjects. Furthermore, after three hours, despite the similar weight loss, elderly men showed higher levels of plasma osmolality and lower plasma volume than that observed in the younger controls. Despite higher degree of dehydration, older men still rated their thirst lower. Plasma osmolality and volume were restored to baseline levels within 30 minutes of drinking in younger subjects but it took 60 minutes for the elderly to restore their osmolality. Plasma volume in the older subjects only returned to baseline values after the 30 minutes of the resting period in $25^{\circ} \mathrm{C}$ suggesting the possibility of agerelated vulnerability to heat stress and resulting thermal dehydration. The diminished thirst response and impaired regulation of osmolality was observed in yet another study by Phillips et al (1991) ${ }^{34}$, where intravascular infusion of hypertonic solution resulted in expansion of plasma volume and increased thirst ratings followed by increased drinking behaviour in the younger controls but resulted in less significant changes in the elderly. Although the changes in baro- and osmoreceptor sensitivities in the elderly have been reported ${ }^{6,8}$, the demonstrated lack of thirst and impaired regulation of plasma volume cannot be simply explained by receptor insensitivity as the levels of arginine vasopressin (ADH) are similar in elderly subjects and younger controls, showing body's attempt to correct hypertonicity and prevent further water loss via urine. While it could be argued that plasma ANP, a known thirst suppressant reported to be increased in the elderly ${ }^{19}$ could be a pathway for hypodipsia, the speculation could be rejected since this hormone could not selectively influence the thirst response and have no impact on AHD. The mechanism of depressed thirst sensation is still unknown, but is most likely to occur due to 
changes in central nervous system and possibly due to changes in oropharyngeal receptors also associated with taste alterations ${ }^{19}$.

\section{Changes in body composition}

Ageing is also associated with changes in body composition. It is well documented that even if there are no variations in body weight, the proportion of fat and fat-free mass changes during the lifetime ${ }^{35}$. A normal trend observed is an increase in body weight and fat mass, following the decrease in weight and fat-free mass at the older age ${ }^{36}$. Hence with time, the proportion of fat-free mass decreases while fat mass increases even in the healthy elderly subjects. Muscle mass is a major determinant of the Resting Metabolic Rate (RMR) and is one of the most metabolically active tissues ${ }^{35}$. Adipose tissue on the other hand stores the energy in a form of lipids and requires minimal amount of energy as it is not as active as other cells. The amount of water in muscle cells is much higher ${ }^{37}$, therefore increased proportion of fat mass also means decline in TBW. This decrease in TBW has been observed in the elderly and it can decline by as much as 4-6 litres by the age of $80^{38}$. Hence in the state of fluid deprivation, the aging body has fewer resources to draw the water from the intracellular fluid compartment and is more likely to become dehydrated.

Despite increased vulnerability due to impairment in water homeostasis, the balance may still be maintained if appropriate hydration takes place. The problem may become evident and quickly escalate when one or more stresses are present ${ }^{4}$. During the states of acute diseases such as infections, vomiting and diarrhoea or during particularly hot weather, these mechanisms may fail and dehydration may occur, even in those appearing very healthy.

\section{Physical disabilities that impair fluid intakes and homeostasis}

The apparent deterioration of homeostatic mechanisms may also be complicated by many disease states and polypharmacy often associated with the elderly. Many age-related conditions may also precipitate increased fluid losses as observed in subjects with Alzheimer's disease due to inappropriate utilization of $\mathrm{ADH}^{6,8}$ or osmotic loss of water with glucose as seen in Diabetes Mellitus ${ }^{39}$. Other conditions, such as renal or congestive heart failure require fluid restriction and/or diuretics; the therapy that should be evaluated frequently but is rarely done $\mathrm{so}^{40}$. Temporary cessation of diuretic therapy should be considered when unusually high amounts of water are being lost either via sweat during fever and exceptionally high temperatures or from gastrointestinal tract from vomiting and diarrhoea ${ }^{13,41}$. Some other conditions such as swallowing difficulties, visual impairment and decline of physical or cognitive status may also influence the ability to obtain the fluids ${ }^{42}$.

\section{CONCLUSION}

Water homeostasis is vital to maintaining optimal health in the elderly population. Considering that elderly should consume the same amounts of fluids as recommended for the younger populations ${ }^{40}$, and the ability to maintain fluid homeostasis is very limited, even in those who appear to be healthy; it becomes increasingly difficult for the aged body to maintain the adequate balance. This explains the relatively high prevalence of fluid and electrolyte imbalances in the elderly ${ }^{43}$. However, considering the age related changes to thirst perception and many disabilities restricting fluid intake; dehydration would be expected to be a more common condition and the elderly, their carers and healthcare professionals need to be appropriately trained and must take action to ensure appropriate water balance is maintained.

\section{REFERENCES}

1. Lecko C. Hydration - the missing part of nutritional care. Nursing Times; 109(26): 1214: (2013)

2. Wolff A, Stuckler D, McKee M. Are patients admitted to hospitals from care homes dehydrated? A retrospective analysis of hypernatraemia and in-hospital mortality. Journal of the Royal Society of Medicine; $0(0)$ : 
1-7: (2015)

3. Himmelstein DU, Jones AA, Woolhandler S. Hypernatremic dehydration in nursing home patients: An indicator of neglect. Journal of the American Geriatrics Society, 31(8): 466-471: (1983)

4. Sheehy C, Perry P, Cromwell S. Dehydration: Biological Considerations, Age-Related Changes, and Risk Factors in Older Adults. Biological Research For Nursing; 1(1): 30-37: (1999)

5. Guyton A. Textbook of medical physiology. 5th ed. Philadelphia: Saunders; (1976).

6. Miller M. Fluid and electrolyte homeostasis in the elderly: Physiological changes of ageing and clinical consequences. Baillière's Clinical Endocrinology and Metabolism; 11(2): 367387: (1997).

7. Hodak SP, Verbalis JG. Abnormalities of water homeostasis in aging. Endocrinology Metabolism Clinics of North America; 34(4): 1031-1046: (2005).

8. Luckey E, Parsa J. Fluid and Electrolytes in the Aged. Archives of Surgery, 138(10): 10551060: (2003).

9. Denny M, Dawson T. Effects of dehydration on body-water distribution in desert kangaroos. American Journal of Physiology, 229(1): 251254: (1975).

10. Nose H, Morimoto T, Ogura K. Distribution of water losses among fluid compartments of tissues under thermal dehydration in the rat. The Japanese Journal of Physiology, 33(6): 1019-1029: (1983).

11. Shanholtzer B, Patterson S. Use of bioelectrical impedance in hydration status assessment: reliability of a new tool in psychophysiology research. International Journal of Psychophysiology, 49(3): 217-226: (2003).

12. Mahowald JM, Himmelstein DU. Hypernatremia in the elderly: Relation to infection and mortality. Journal of the American Geriatrics Society; 29(4): 177-180: (1981).

13. Thomas DR, Cote TR, Lawhorne L, Levenson SA, Rubenstein LZ, Smith DA, et al. Understanding clinical dehydration and its treatment. Journal of the American Medical Directors Association; 9(5): 292-301: (2008).
14. Mange $\mathrm{K}$, Matsuura D, Cizman B, Soto $\mathrm{H}$, Ziyadeh FN, Goldfarb S, et al. Language guiding therapy: the case of dehydration versus volume depletion. Annals of Internal Medicine; 127(9):848: (1997).

15. Noakes T. The hyponatremia of exercise. International Journal of Sport Nutrition and Exercise Metabolism; 2(3) :205-228: (1992).

16. Chorro I, Sant L, Martin M. Hyponatremia, cerebral edema and brain death in a MDMA acute intoxication. Medicina Clínica; 124(5): 198-198: (2005).

17. Anonymous. 'Autopsy Finds Signs of Water Intoxication in Radio Contestant's Death' Wayback Machine; 2007. Available at https:// web.archive.org/web/20070926234242/ http://www.news10.net/display story. aspx? storyid=23350 2007, last accessed 21 August 2016

18. Brooks, L. 'Pledge's family pushes for antihazing law'. The Orion; 2005 available at: www.theorion.com Last accessed 21 August, 2016

19. McNab, H. 'I just never thought that water could kill you': The woman who drank 5 LITRES of water as part of a detox diet but ended up in a COMA after poisoning herself. Mail Online; 2014. Available at: http://www. dailymail.co.uk/news/article-2786563/l-justnever-thought-water-kill-Meet-woman-drank5L-water-detox-ended-COMA-poisoningherself.html\#ixzz4I3vLwK4D. Last accessed: 21 August 2016.

20. Noakes TD. Commentary: The role of hydration in health and exercise. British Medical Journal; 345(7866): 28-28: 2012

21. Begum MN, Johnson CS. A review of the literature on dehydration in the institutionalized elderly. e-SPEN, the European e-Journal of Clinical Nutrition and Metabolism; 5(1) :e47e53: (2010).

22. Mentes J, Wakefield B, Culp K. Use of a urine color chart to monitor hydration status in nursing home residents. Biological Research For Nursing; 7(3): 197-203: (2006).

23. Rolls BJ, Phillips PA, Phil D. Aging and disturbances of thirst and fluid balance. Nutrition Reviews; 48(3): 137-144: (1990).

24. Erkert J.D. Dehydration in the elderly. $J A m$ Acad Physician Assist; 1:261-269;(1988) 
25. Phillips PA, Rolls BJ, Ledingham JG, Morton JJ. Body fluid changes, thirst and drinking in man during free access to water. Physiology \& Behavior, 33(3): 357: (1984).

26. Rittig S, Knudsen U, Norgaard J, Pedersen $\mathrm{E}$, Djurhuus J. Abnormal diurnal rhythm of plasma vasopressin and urinary output in patients woth enuresis. American Journal of Physiology, 256(4): F664-F671: (1989).

27. Asplund R. Nocturia, nocturnal polyuria, and sleep quality in the elderly. Journal of Psychosomatic Research; 56(5): 517-525: (2004).

28. Donahue J, Lowenthal D. Nocturnal polyuria in the elderly person. The American Journal of the Medical Sciences; 314(4): 232-238: (1997).

29. Rittig S, Knudsen U, Norgaard J, Pedersen $\mathrm{E}$, Djurhuus J. Abnormal diurnal rhythm of plasma vasopressin and urinary output in patients woth enuresis. American Journal of Physiology, 256(4): F664-F671: (1989).

30. Asplund R, Aberg H. Health of the elderly with regard to sleep and nocturnal micturition. Scandinavian Journal of Primary Health Care; 10(2): 98: (1992).

31. Asplund $\mathrm{R}$, Aberg $\mathrm{H}$. Diurnal variation in the levels of antidiuretic hormone in the elderly. Journal of Internal Medicine; 229(2) :131: (1991).

32. Crowe MJ, Forsling ML, Rolls BJ, Phillips PA, Ledingham JGG, Smith RF. Altered water excretion in healthy elderly men. Age and Ageing; 16(5): 285-293: (1987).

33. Miescher E, Fortney SM. Responses to dehydration and rehydration during heat exposure in young and older men. American Journal of Physiology; 257(5): 26/5: (1989).

34. Phillips PA, Bretherton M, Johnston Cl, Gray L. Reduced osmotic thirst in healthy elderly men. American Journal of Physiology; 261(1 Pt 2): R166-171: (1991).
35. St-Onge M-, Gallagher D. Body composition changes with aging: The cause or the result of alterations in metabolic rate and macronutrient oxidation? Nutrition; 26(2): 152-155: (2010).

36. Going S, Williams D, Lohman T. Aging and body composition: biological changes and methodological issues. Exercise and Sport Sciences Reviews; 23: 411: (1995).

37. Armstrong LE. Hydration Assessment Techniques. Nutrition Reviews; 63(Supplement 1): 40-54: (2005).

38. Gille D. Overview of the physiological changes and optimal diet in the golden age generation over 50. European Review of Aging and Physical Activity; 7(1): 27-36: (2010).

39. Chiasson J, ArisJilwan N, Belanger R, Bertrand $\mathrm{S}$, Beauregard $\mathrm{H}$, Ekoe J, et al. Diagnosis and treatment of diabetic ketoacidosis and the hyperglycemic hyperosmolar state. CMAJ Canadian Medical Association Journal; 168(7): 859-866: (2003).

40. Ferry M, Dal Canton A, Manz F, Armstrong L, Sawka M, Ritz P, et al. Strategies for ensuring good hydration in the elderly. Nutrition Reviews; 63(6): S22-29: (2005).

41. Perren A, Markmann M, Merlani G, Marone C, Merlani P. Fluid balance in critically ill patients. Should we really rely on it? Minerva Anestesiologica; 77(8): 802-811: (2011).

42. Schols JM, De Groot CP, van der Cammen TJ, Olde Rikkert MG. Preventing and treating dehydration in the elderly during periods of illness and warm weather. The Journal of Nutrition Health and Aging; 13(2): 150-157: (2009).

43. Arinzon Z, Feldman J, Peisakh A, Zuta A, Berner Y. Water and sodium disturbances predict prognosis of acute disease in long term cared frail elderly. Archives of Gerontology and Geriatrics; 40(3): 317-326: (2005). 